\title{
Reactive oxygen species, dietary restriction and neurotrophic factors in age-related loss of myenteric neurons
}

\author{
C. Thrasivoulou, ${ }^{1}$ V. Soubeyre, ${ }^{1}{ }^{*} \mathrm{H}$. Ridha, ${ }^{1}$ \\ D. Giuliani, ${ }^{2}$ C. Giaroni, ${ }^{2}$ G. J. Michael, ${ }^{3}$ \\ M. J. Saffrey ${ }^{4}$ and T. Cowen ${ }^{1}$ \\ ${ }^{1}$ Department of Anatomy and Developmental Biology, University \\ College London, Royal Free Campus, London NW3 2PF, UK \\ ${ }^{2}$ Clinical and Applied Pharmacology Centre, University of Insubria \\ and University of Pavia, Italy \\ ${ }^{3}$ Neuroscience Centre, Queen Mary, University of London, London \\ E1 2AT, UK \\ ${ }^{4}$ Department of Biological Sciences, Open University, Milton Keynes \\ MK7 6AA, UK
}

\section{Summary}

We have studied the mechanisms underlying nonpathological age-related neuronal cell death. Fifty per cent of neurons in the rat enteric nervous system are lost between 12 and 18 months of age in ad libitum (AL) fed rats. Caloric restriction (CR) protects almost entirely against this neuron loss. Using the ROS-sensitive dyes, dihydrorhodamine (DHR) and 2-[6-(4'-hydroxy)phenoxy3H-xanthen-3-on-9-yl]benzoic acid (HPF) in vitro, we show that the onset of cell death is linked with elevated intraneuronal levels of reactive oxygen species (ROS). Treatment with the neurotrophic factors NT3 and GDNF enhances neuronal antioxidant defence in CR rats at 1215 months and 24 months but not in adult or aged AL-fed animals. To examine the link between elevated ROS and neuronal cell death, we assessed apoptotic cell death following in vitro treatment with the redox-cycling drug, menadione. Menadione fails to increase apoptosis in 6-month neurons. However, in 12-15mAL fed rats, when age-related cell death begins, menadione induces a 7- to 15-fold increase in the proportion of apoptotic neurons. CR protects age-matched neurons against ROS-induced apoptosis. Treatment with neurotrophic factors, in particular GDNF, rescues neurons from menadione-induced cell death,

\footnotetext{
Correspondence

T. Cowen, PhD, Department of Anatomy and Developmental Biology, University College London, Royal Free Campus, Rowland Hill St., London NW3 2PF, UK. Tel.: (0)207 830 2181; fax: (0)207 472 6763;

e-mail: tcowen@medsch.ucl.ac.uk

*Present address: Neurobiologie des Processus Adaptatifs (UMR 7102), Université Pierre et Marie Curie (Paris VI), Bât B, 6e étage, Case 12, 9, quai St Bernard, 75005 Paris, France
}

Accepted for publication 13 March 2006 but only in 12-15mCR animals. We hypothesize that CR enhances antioxidant defence through neurotrophic factor signalling, thereby reducing age-related increases in neuronal ROS levels and in ROS-induced cell death. Key words: aging; antioxidant defence; apoptosis; cell death; dietary restriction; myenteric neurons; neurotrophic factors; reactive oxygen species.

\section{Introduction}

Relatively little is known about the mechanisms which underlie nonpathological age-related neurodegeneration. Neither the nature nor extent of this phenomenon is well established. Studies of mammalian central (Morrison \& Hof, 1997; Turlejski \& Djavadian, 2002; Baquet et al., 2004) and peripheral (Cowen \& Gavazzi, 1998; Cowen et al., 2003) neurons demonstrate that age-associated degenerative changes only occur in select groups of neurons such as the cholinergic neurons of the basal forebrain and the sympathetic neurons projecting to cerebral blood vessels and pineal gland. Previous studies have shown that around $50 \%$ of the neurons of the myenteric plexus in the gut of rodents (Johnson et al., 1998; Cowen et al., 2000), guinea pigs (Gabella, 1989) and humans (Porter et al., 1996) are lost during aging. While in rats neurons expressing choline acetyl transferase are vulnerable while those expressing nitric oxide synthase are protected (Cowen et al., 2000), the reasons for this selective vulnerability in different regions of the nervous system are poorly understood.

Age is the biggest risk factor for several of the more common neurodegenerative diseases, including Alzheimer, Parkinson and motor neuron diseases. Damage by reactive oxygen species (ROS) is implicated in nonpathological brain aging processes (Poon et al., 2004; Lau et al., 2005) as well as in all of these chronic disorders (Schapira, 1998; Jung et al., 2001; Nunomura et al., 2001; Huang et al., 2004). Although there is little concrete evidence, the implication of these observations is that common mechanisms involving ROS may underpin pathological as well as nonpathological age-related neurodegeneration.

Neurons are considered particularly vulnerable to free radical damage because of their large size, high level of metabolic activity and relatively poor antioxidant defence (Sohal \& Weindruch, 1996). Survival of adult and aged neurons appears to be in some way related to the capacity to resist ROS-induced damage (Finkel \& Holbrook, 2000). Previous evidence of age-related vulnerability of enteric neurons (Phillips et al., 2003) does not provide a link with ROS sensitivity. Calcium dyshomeostasis is a key event in neuronal aging (Bu et al., 2003) and in Alzheimer 
disease (LaFerla, 2002) which have been linked to altered mitochondrial ROS generation in old age (Mattson \& Liu, 2002). The calcium-binding proteins, calretinin and calbindin, present in many central and peripheral neurons including neurons of the myenteric plexus (Abalo et al., 2005), are thought to protect neurons against calcium dyshomeostasis and may therefore allow the identification of neurons that are selectively protected against ROS-induced damage.

Caloric restriction (CR) extends lifespan in organisms as diverse as yeasts, worms, flies and mammals by enhancing the capacity for stress resistance (Sohal \& Weindruch, 1996). Furthermore, CR enhances plasticity in the aging brain (Mattson et al., 2002), increases expression of an anti-apoptotic repressor in the cerebral cortex of aging rats (Shelke \& Leeuwenburgh, 2003), and rescues neurons of the rat enteric nervous system (ENS) from age-related cell death (Cowen et al., 2000). We hypothesize that the effects of diet on neuronal survival are mediated by altered capacity to regulate ROS.

Neurotrophic factor signalling is crucial to neuronal survival and differentiation during development (Miller \& Kaplan, 2001). A novel role for neurotrophins in regulating ROS has recently been demonstrated in developing sympathetic neurons (Dugan et al., 1999), in PC 12 cells (Mills et al., 1998) and in an animal model of Parkinson's disease (Salinas et al., 2003). Because CR has been shown to influence neurotrophin-mediated plasticity in the CNS (Prolla \& Mattson, 2001; Nicoletti et al., 2005), we hypothesize that $C R$ might enable neurons to resist age-related cell death by enhancing neurotrophic factor-mediated antioxidant defence.

Our aims were therefore (i) to confirm protection by dietary restriction against age-related loss of myenteric neurons and to determine whether neurons expressing calcium-binding proteins were selectively protected against age-related cell death; (ii) to find out if age- and diet-related alterations in intracellular ROS levels are associated with neuronal cell death in the ENS; (iii) to discover whether elevated ROS levels might induce apoptotic cell death in enteric neurons; and (iv) to demonstrate a possible role for neurotrophic factors in regulating antioxidant defence and resistance to ROS-induced neuronal cell death.

\section{Results}

\section{Effects of dietary restriction on age-related loss of myenteric neurons}

Counts of PGP 9.5-stained neurons confirm previous data (Cowen et al., 2000) by showing an age-related loss of $51 \%$ of neurons $(P<0.001)$ in $24 \mathrm{mAL}$ animals (Fig. 1A). CR animals show a much smaller loss of neurons over $24 \mathrm{~m}(22 \% ; P<0.05)$ indicating, like our previous data, substantial protection by dietary restriction. Examination of the timescale of neuron loss reveals that at 12 months, numbers of neurons are not significantly different from those in 6-month rats and there are no differences between $A L$ and $C R$ groups (Fig. 1A). However, by 13 months there are $24 \%(P<0.05)$ fewer neurons in the $A L$
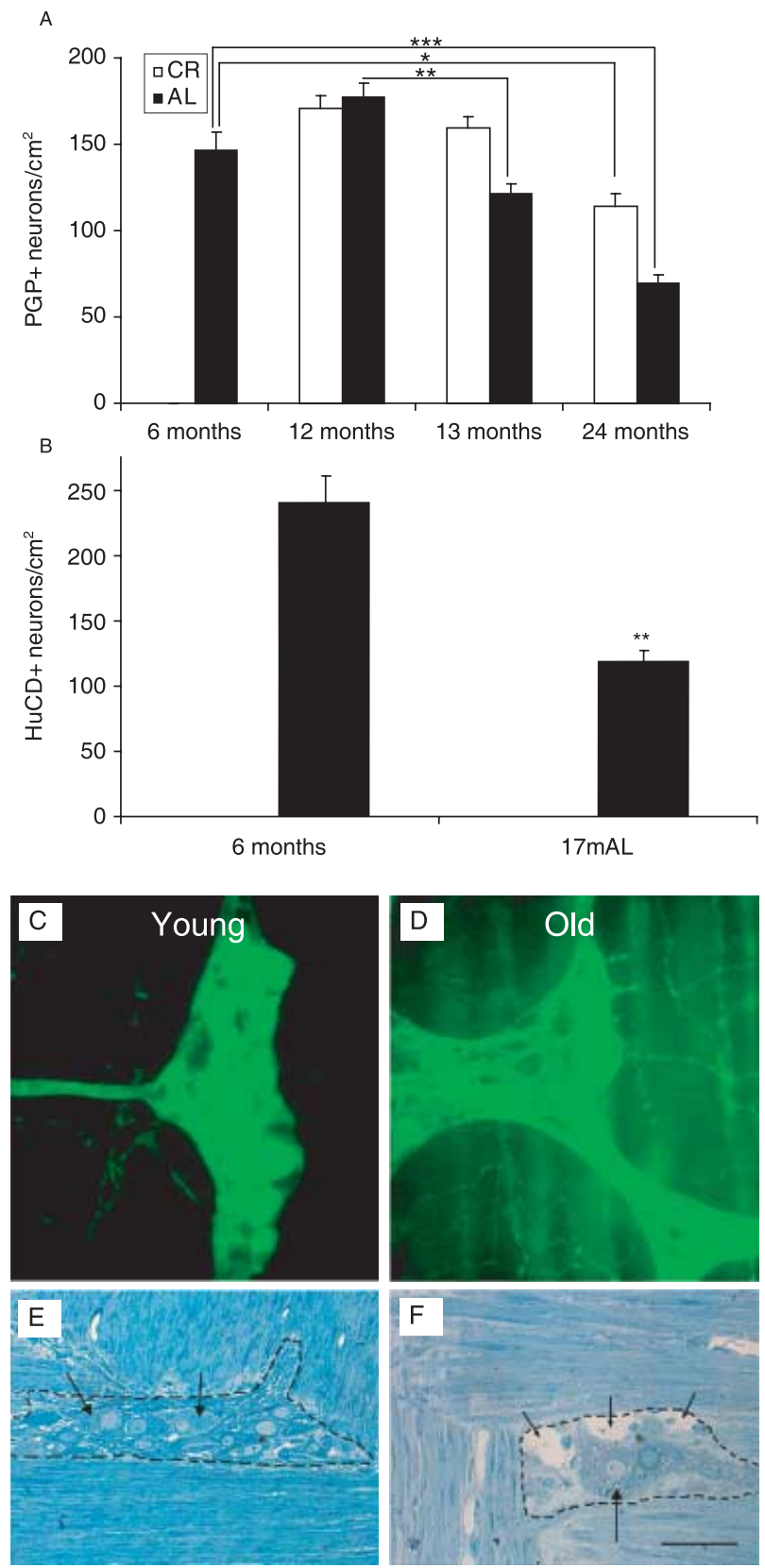

Fig. 1 Total numbers of myenteric neurons. (A) Counts of PGP-

immunoreactive neurons. The histogram shows significant losses of neurons, commencing at 12-13 months of age in AL-fed animals and reaching a peak at 24 months ( $A L ; 51 \%$ ). There is a much smaller but statistically significant $(P<0.05)$ reduction seen in $24 \mathrm{mCR}$ samples, demonstrating substantial protection by $C R$ against age-related cell death in myenteric neurons.

(B) Counts of HuCD-immunoreactive neurons. The histogram shows loss of neurons in 17-month AL-fed, confirming that PGP 9.5 labelling gives a reliable estimate of age- and diet-related cell loss. However, total neuron numbers were significantly $(P<0.01)$ higher in the HuC/D-stained samples. (C-F) Photomicrographs of 6-month (C,E) and 24-month AL (D,F) PGPimmunostained whole-mounts $(C, D)$ and toluidine blue-stained $1-\mu m$ resin sections ( $E, F)$. Neurons in myenteric ganglia (dotted lines) are indicated by large arrows. Note the large spaces in the ganglia from 24-month AL rats (small arrows) visible in D and $F$ but absent in the 6-month samples $(C, E)$, indicating where neuron cell loss has occurred. $\mathrm{n}$ numbers: $\mathrm{A}: 6$ months $(n=15) ; 12 \mathrm{mCR}(n=4) ; 12 \mathrm{mAL}(n=4) ; 13 \mathrm{mCR}(n=8) ; 13 \mathrm{mAL}(n=12)$; $24 \mathrm{mCR}(n=15) ; 24 \mathrm{mAL}(n=14) ; \mathrm{B}: 6$ months $(n=6) ; 17$ months $(n=3)$. Scale bar $=50 \mu \mathrm{m}$. 
compared to $C R$ groups and this number is significantly lower than either of the 12 months groups (13mAL vs. $12 \mathrm{mCR}, P<0.05$; $13 \mathrm{mAL}$ vs. $12 \mathrm{mAL}, P<0.01$ ). As reported previously (Cowen et al., 2000), neuron loss is complete by $16-18$ months (when corrected for gut size) in $\mathrm{AL}$-fed rats. Whilst these data show similar levels of loss of neurons with HuC/D at $17 \mathrm{mAL}$ ( $50 \%$ ) with those shown in Cowen et al. (2000), the PGP 9.5 data only show a partial, but significant loss, at the $13 \mathrm{mAL}$ age group studied. Counts are corrected for changes in gut size to ensure that alterations are due to cell loss rather than redistribution of neurons. However, our measurements reveal no significant effect of diet or age on the overall dimensions of the small intestine (Table 1).

As a result of a recent study evaluating pan-neuronal markers in the rat myenteric plexus (Phillips et al., 2004), tissues were taken from small groups of 3-month and $17 \mathrm{mAL}$ animals and immunolabelled for HuC/D, which was shown to be one of the most reliable pan-neuronal markers for rodent myenteric neurons. Neuron counts made on HuC/D-labelled preparations demonstrated significantly $(P<0.01)$ higher neuron numbers compared to PGP 9.5, confirming previous reports that PGP 9.5 does not stain all neurons in the gut (Young et al., 2003). HuC/D, however, demonstrated a comparable loss of neurons (50\%; $P<0.01$; Fig. 1B) in $17 \mathrm{mAL}$ fed animals to that shown previously using PGP 9.5 at 16 and 24 months in AL-fed rats (Cowen et al., 2000). The $50 \%$ loss of neurons seen with HuC/D at 17 months is similar to the extent of loss of PGP 9.5-positive neurons seen at 24 months (Fig. 1A,B), supporting the view that neuron loss in aging AL-fed rats is largely complete by 17-18 months.

Loss of immunolabelling may indicate reduced binding of the antibodies rather than neuron loss. To examine this possibility, we studied parallel samples from $24 \mathrm{mAL}$ and 6-month rats following PGP staining of whole mounts and toluidine blue

Table 1 Age- and diet-related changes in areal dimensions $\left(\mathrm{cm}^{2}\right)$ of small intestine

\begin{tabular}{ll}
\hline 6 months & $148.1 \pm 5.0$ \\
\hline $13 \mathrm{mCR}$ & $144.8 \pm 13.5$ \\
$13 \mathrm{mAL}$ & $128.7 \pm 6.7$ \\
$24 \mathrm{mCR}$ & $134.2 \pm 9.4$ \\
$24 \mathrm{mAL}$ & $158.4 \pm 3.7$ \\
\hline
\end{tabular}

There are no statistically significant differences between any of these measurements (i.e. there is no significant change in gut dimensions with age or diet). staining of $1 \mu \mathrm{m}$ resin sections (Fig. 1C-F). Both methods reveal clearly defined spaces surrounded by enteric glia in the ganglia of $24 \mathrm{mAL}$ samples which are not present in 6-month samples. Overall, these results confirm that age-related neuron loss has indeed taken place and that dietary restriction protects myenteric neurons against age-related loss.

\section{Selective vulnerability in subpopulations of myenteric neurons}

Calcium dyshomeostasis is a key event in neuronal aging (Bu et al., 2003). We therefore investigated whether neurons containing the calcium-binding proteins, calretinin and calbindin, were particularly protected compared to the general neuron population in aging rats. Calretinin-positive neurons represented a significant (25\%) proportion of the total population of myenteric neurons and exhibited similar small but nonsignificant losses at 17 months of age in preparations from both AL- or CR-fed animals (Fig. 2A).

Calbindin-positive neurons formed a much smaller proportion of the total $(2.5 \%)$ (compare Fig. 2A,B). Numbers were reduced by $62 \%(P<0.01)$ in the $17 \mathrm{mAL}$ fed group indicating that these neurons were selectively vulnerable during aging, while $C R$ at the same age demonstrated almost complete protection of calbindin-positive neurons (Fig. 2B). These results suggest that while the calcium-buffering capacity of calretinin may be a factor in protecting these neurons against age-related calcium dyshomeostasis and death, calbindin is not associated with a similar protective effect. Dietary restriction is shown to be strongly neuroprotective in the case of the calbindin-positive population of myenteric neurons.

\section{ROS levels in myenteric neurons}

Initial comparisons revealed a visually obvious elevation of ROS levels in 24-month compared to 6-month myenteric neurons using the ROS-sensing dye, DHR (Fig. 3A,B). We therefore made systematic comparisons of neuronal ROS levels between the different experimental groups. In vitro preparations of 6-month rat myenteric neurons exhibit different rates of increase in DHR fluorescence during the period of incubation, which peaks around $2 \mathrm{~h}$ (data not shown). This timescale was therefore used in all subsequent experiments. Comparison of ROS levels using DHR fluorescence in preparations from 6-month and from 12- to
Fig. 2 Numbers of calretinin- (A) and calbindin- (B) immunoreactive myenteric neurons. Note the lack of significant change in calretinin + neurons between 6 and 17 months of age or with $\mathrm{AL}$ feeding which contrasts with the age-related loss of over $50 \%$ of calbindin + neurons in the $17 \mathrm{mAL}$ group $(P<0.01)$ which is fully rescued in age-matched $C R$ animals. $n$ numbers: $A$ : 6 months $(n=4) ; 17 \mathrm{mCR}(n=3) ; 17 \mathrm{mAL}(n=4) ; \mathrm{B}$ : 6 months $(n=5) ; 17 \mathrm{mCR}(n=3) ; 17 \mathrm{mAL}(n=6)$.

\section{A}

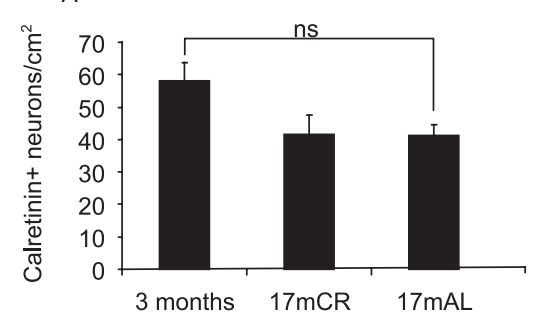

B

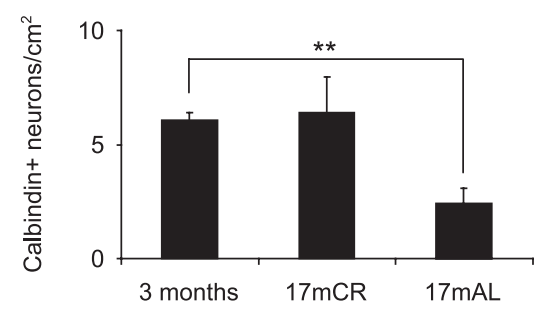



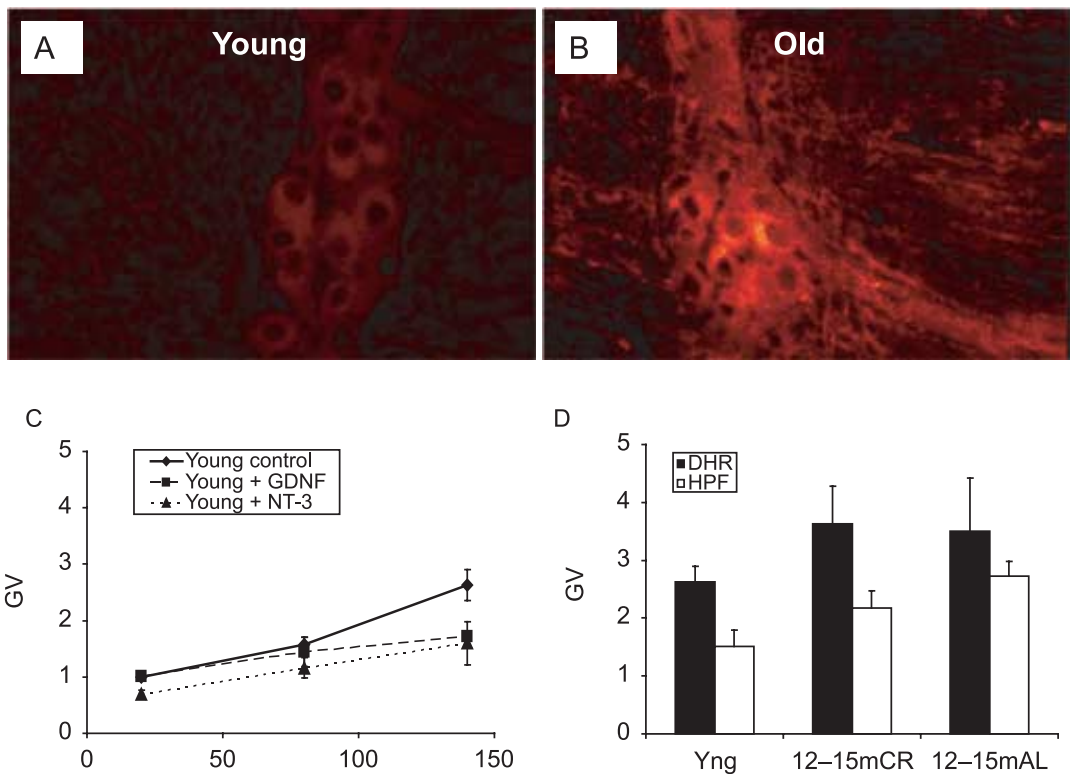

D

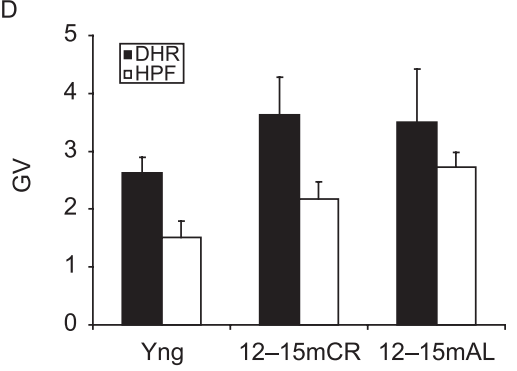

$E$

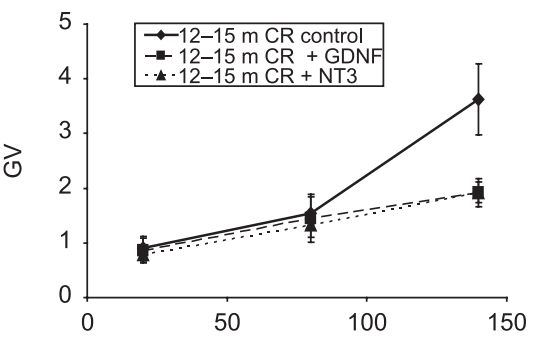

F

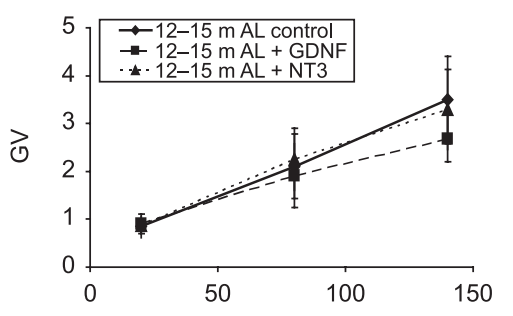

G

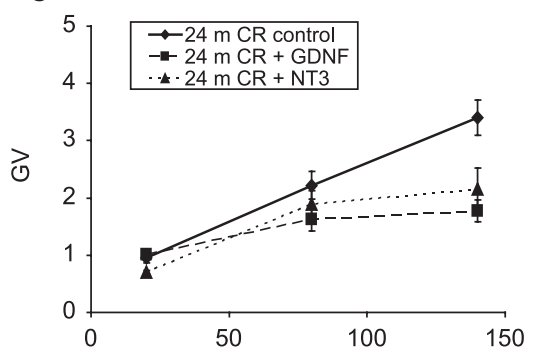

$\mathrm{H}$

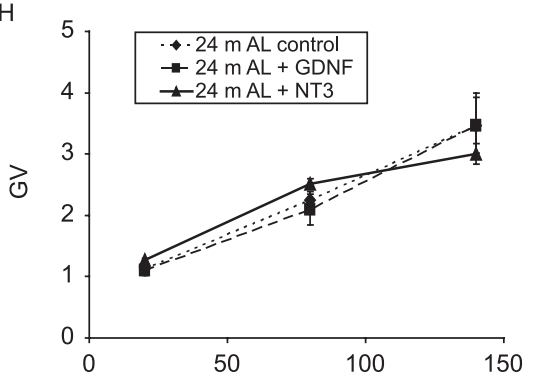

Fig. 3 ROS generation in myenteric neurons. Photomicrographs of 6-month (A) and $24 \mathrm{mAL}(B)$ whole mount preparations of myenteric neurons showing ROS-induced DHR fluorescence generated following $2 \mathrm{~h}$ of incubation. Note the substantial increase in brightness of cytoplasmic fluorescence in the $24 \mathrm{mAL}$ preparation. $(\mathrm{C}-\mathrm{H})$ Graphs showing extent and timescale of free radical generation $(\mathrm{GV}=$ mean grey value of $\mathrm{DHR}$ fluorescence intensity) in enteric neurons, with and without treatment with the neurotrophic factors, NT3 and GDNF, from 6 months $(C ; n=10)$, $12-15 \mathrm{mCR}(\mathrm{E} ; n=4)$ and $\mathrm{AL}(\mathrm{F} ; n=4)$ and $24 \mathrm{mCR}$ ( $\mathrm{G} ; n=4)$ and $\mathrm{AL}(\mathrm{H} ; n=12)$ rats over $2 \mathrm{~h}$ of incubation. (D) Graph showing fluorescence intensity (mean GV) in 6 months, 12-15mCR and 12-15mAL preparations following $2 \mathrm{~h}$ of incubation in DHR (as C) or in the $\mathrm{OH}$-sensitive probe, HPF. Note the similar age-related increase in HPF fluorescence compared to that shown by DHR. Both groups, $(n=3)$. 15-month CR rats shows increased levels of free radical generation with age (Fig. 3C,E). Samples from 12- to 15-month AL rats, where neuron loss has begun (see Fig. 1), show a slightly sharper rate of increase of ROS levels assessed after $1 \mathrm{~h}$ of incubation compared to CR specimens of the same age (Fig. 3E,F), suggesting that $C R$ may partially protect neurons against dietinduced elevation of free radical generation. The (nonsignificant) increases in ROS after 2 h of incubation are summarized in Fig. 3D.

Comparison of DHR fluorescence levels in preparations from 24-month animals indicates a significant effect of age on fluorescence levels compared to 6-month neurons after $1 \mathrm{~h}$ of incubation [Fig. 3; comparing C (6 months; $1.58 \pm 0.169)$ and $\mathrm{G}(24 \mathrm{mCR} ; 2.22 \pm 0.066)$, and C (6 months) and H (24mAL; $2.24 \pm 0.148$ ); (both $P<0.02$ )], suggesting that increasing age correlates with higher levels of intrinsic neuronal ROS. There is no significant difference between the extent or rate of increase in free radical levels in $24 \mathrm{mAL}$ compared to $24 \mathrm{mCR}$ groups indicating that the neuroprotective effect of dietary restriction is exhausted by 24 months (the mean lifespan of Sprague Dawley rats).

DHR fluorescence generated by the oxidation of dihydrorhodamine to rhodamine 123 may be influenced by mitochondrial membrane potential (Hempel et al., 1999) as well as by other age-associated variables such as ferric iron and cytochrome c. In order to confirm the effects of age and diet on neuronal ROS levels, we assessed neuronal levels of fluorescence using the ROS-sensing dye, HPF (2-[6-(4'-hydroxy)phenoxy-3H-xanthen-3on-9-yl]benzoic acid) (Setsukinai et al., 2003), in myenteric neurons from young and old AL-fed animals (Fig. 3D). HPF is specifically activated by $\mathrm{OH}$, derived principally from superoxide (Setsukinai et al., 2003; Ooe et al. 2005). HPF fluorescence, like 
DHR, increases with incubation time, peaking after 2-3 h (data not shown). HPF demonstrates an age-related increase in neuronal ROS in the 12- to 15-month samples which is slightly, but not significantly, greater in the AL group. These differences are similar to those seen with DHR, confirming that DHR fluorescence provides a reliable measure of ROS levels in myenteric neurons. Because HPF fluorescence is specifically induced by superoxide-derived $\mathrm{OH}^{\bullet}$ it is likely that mitochondrial ROS provide the majority of the free radicals assessed in our experiments.

\section{Neurotrophic factors in antioxidant defence}

Because of evidence that neurotrophic factors contribute to antioxidant defence in early postnatal neurons (Dugan et al., 1999), we tested the capacity of GDNF and NT3, with known survival-promoting roles in myenteric neurons (Chalazonitis et al., 2001; Busciglio et al., 2002), to reduce ROS levels in these neurons from adult rats. Treatment of in vitro preparations of enteric neurons from 6-month rats with $100 \mathrm{ng} \mathrm{mL} \mathrm{m}^{-1}$ of GDNF or NT3 results in significantly lower levels of neuronal ROS compared to untreated preparations after $2 \mathrm{~h}$ of incubation (Fig. 3C; GDNF: $P<0.01$; NT3: $P<0.05$ ). In contrast, treatment with NGF or IGF-1 [with known antioxidant effects in other groups of neurons (Dugan et al., 1999) and cell types (Jallali et al., 2005)], has no effect on ROS levels (data not shown).

Comparisons of the effects of neurotrophic factors on ROS levels in 12- to 15-month animals (Fig. 3E,F) show that in CR preparations, ROS are significantly reduced at the 2 -h time point after treatment with NT3 and GDNF (both, $P<0.05$ ). In contrast, neither factor has a significant effect on ROS levels in preparations from 12- to 15-month AL rats. Thus CR appears to enhance neurotrophin-mediated antioxidant mechanisms around the age at which cell loss occurs in vivo. In the $24 \mathrm{mCR}$ group, treatment with neurotrophic factors suppresses ROS levels significantly (Fig. 3G,H; GDNF: $P<0.001$; NT3: $P<0.05$ ) but not in the AL preparations, resembling the effects seen at 12-15 months. It is worth noting that these differential effects, like the onset of neuron cell death, are already established at the relatively young age of 12-15 months (i.e. well before the onset of senescence). Our results therefore show that age enhances levels of ROS generation, while age and diet, respectively, inhibit and enhance neurotrophic factor-mediated antioxidant defence mechanisms, in adult and aged neurons.

\section{ROS-induced neuronal cell death}

In order to discover whether ROS contribute to age- and dietinduced neuronal cell death, we examined the effect of treatment with menadione (a drug which accelerates mitochondrial ROS generation by redox cycling) on the incidence of apoptotic cell death in myenteric neurons. We chose to focus on the period up to and including the early stages of age-related neuron loss in the myenteric plexus (i.e. 12-15 months of age). The increase in the TUNEL response to menadione was timedependent, reaching a maximum after $4 \mathrm{~h}$ of incubation (data
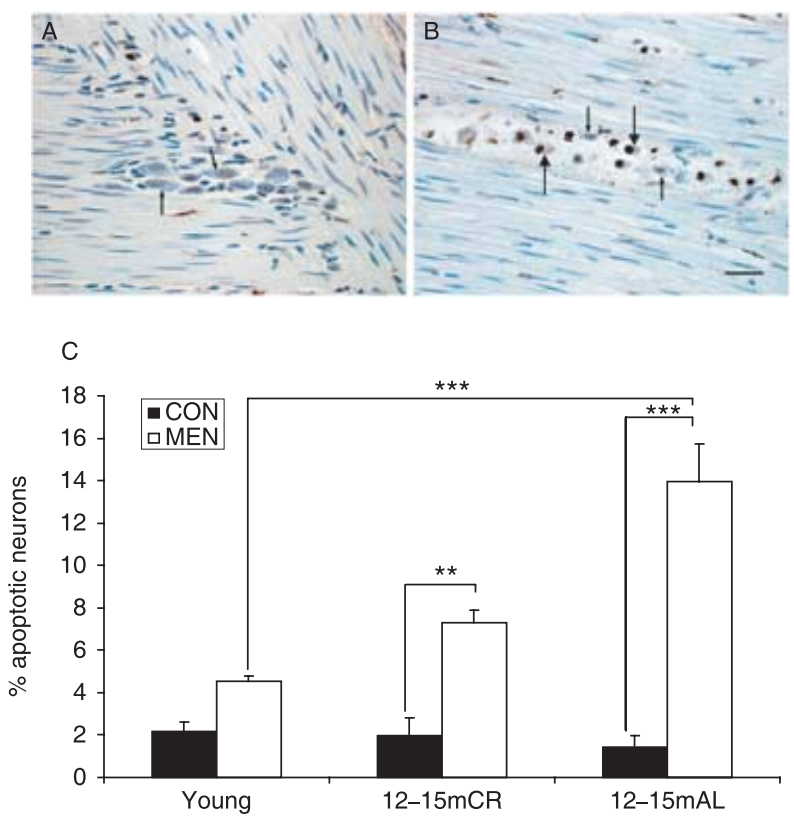

Fig. 4 ROS-induced apoptotic neuron cell death: TUNEL-stained cryosections of 6-month (A) and 12-15mAL (B) myenteric neurons following $4 \mathrm{~h}$ incubation with menadione. Toluidine blue stained neurons (small arrows, $A$ and $B$ ) are healthy; neurons showing brown TUNEL-positive nuclei (large arrows, B only), some of which are fragmented or with crenellated outlines, are undergoing apoptosis. The histogram (C) shows the proportion of TUNELpositive neurons from the different experimental groups following treatment with menadione. Note the neuroprotective effect of CR after treatment with menadione at $12-15$ months. Six months groups $(n=10) ; 12-15 \mathrm{mCR}$ groups $(n=6) ; 12-15 \mathrm{mAL}$ con $(n=4) ; 12-15 \mathrm{mAL}+\operatorname{men}(n=8)$.

not shown). Menadione treatment for $4 \mathrm{~h}$ in vitro produced a visually obvious increase in TUNEL-positive neurons in preparations from 12- to 15-month $A L$ animals compared to those from 6-month animals (Fig. 4A,B). In addition, other cells including smooth muscle, endothelial and glial cells can be seen to exhibit significant vulnerability to ROS-induced apoptosis. Counts of TUNELpositive neurons and visual examination of nuclear morphology reveal that after $4 \mathrm{~h}$ of incubation in culture medium without menadione, sparse and unaltered numbers of apoptotic profiles were present in all experimental groups (Fig. 4C). Treatment with menadione induces a small but nonsignificant increase in apoptotic profiles in 6 months preparations compared to untreated controls (Fig. 4C). In contrast, the proportion of apoptotic neurons after menadione treatment increases 10 -fold $(P<0.001)$ in the 12-15mAL group; a similar increase in apoptotic neurons after menadione was seen in samples from $24 \mathrm{mAL}$ animals (data not shown). Interestingly, CR protects 12- to 15-month neurons from menadione-induced cell death, reducing the proportion of apoptotic neurons significantly compared to the $\mathrm{AL}$ group (Fig. 4C; $P<0.001$ ). Menadione-induced apoptosis is apparently higher in the $12-15 \mathrm{mCR}$ group compared to 6-month samples, but the difference is not statistically significant. Examination of nuclear morphology revealed an increase of fragmented and crenellated nuclei in both of the 12- to 15-month groups, suggesting strongly that at least a proportion of these neurons 
were dying apoptotically. Thus, age and ad libitum feeding enhance vulnerability to ROS-induced death of myenteric neurons.

Next we wanted to find out whether menadione-induced death of neurons could be ameliorated by in vitro treatment with antioxidants and by neurotrophic factors. Preparations from 6-month and from 12- to 15-month $\mathrm{AL}$ and $\mathrm{CR}$ animals were treated with menadione as before, following pretreatment with the synthetic antioxidant carboxyfullerene also known as C3 (Dugan et al., 1997) or with the neurotrophic factors GDNF or NT3 (Fig. 5A-C). Fullerene compounds react avidly with free radicals and are regarded as 'radical sponges'. The trimalonic acid derivative of fullerene, C3, is a synthetic water-soluble compound that has been found to be an effective antioxidant both in vivo and in vitro (Tsao et al., 2002). Preliminary experiments using a 'Live-Dead' assay (Molecular Probes Inc., Eugene, OR, USA) demonstrated improved imaging and sampling compared to the TUNEL method and this was therefore used for these experiments. The effects of menadione on cell death are qualitatively similar using this assay compared with TUNEL staining in the young and 12-15mAL groups. However, menadione induces a greater extent of cell death as measured by this method compared with TUNEL staining in the $12-15 \mathrm{mCR}$ group. We speculate that this is because the Live-Dead assay demonstrates ethidium staining in compromised cells not yet committed to apoptosis, i.e. still capable of being rescued (see below).

C3 reduces the extent of cell death very markedly in all groups (Fig. 5). Interestingly, the rescue effect of C3 is greatest in the $12-15 \mathrm{mCR}$ group (96\% and $P<0.001$, compared to $80 \%$ and $P<0.01$ for $12 \mathrm{mAL}$ ). GDNF pretreatment produced no significant effect on cell death in young or in $12-15 \mathrm{mAL}$ preparations. However, GDNF exerted significant protection (45\%; $P<0.01$ ) in the $12-15 \mathrm{mCR}$ group. Pretreatment with NT3 gives small but nonsignificant rescue from menadione-induced cell death in all groups, but with no difference between 12 and $15 \mathrm{mAL}$ and $C R$ animals. Because of the greater rescue effects of $C 3$ and GDNF in the 12-15mCR group, we speculate that the LiveDead assay demonstrates ethidium staining in compromised cells not yet committed to apoptosis, i.e. still capable of being rescued. The presence of small numbers of cells staining for both calcein and ethidium supports this hypothesis. Our results suggest that at the stage of life when neurons become vulnerable to age-related cell death, caloric restriction enhances the protective effect of neurotrophic factors against ROS-induced cell death. The lack of protective effect of GDNF at the young stage may indicate that the young enteric neurons already obtain a maximal supply of the endogenous growth factor, or have other ways of inducing maximal antioxidant defence. Alternatively it is possible that the antioxidant defensive role of GDNF increases during adult life. Ongoing studies are examining these alternatives.

\section{Discussion}

In this study, the mechanisms underlying age-related loss and cell death of rat myenteric neurons have been examined. At the
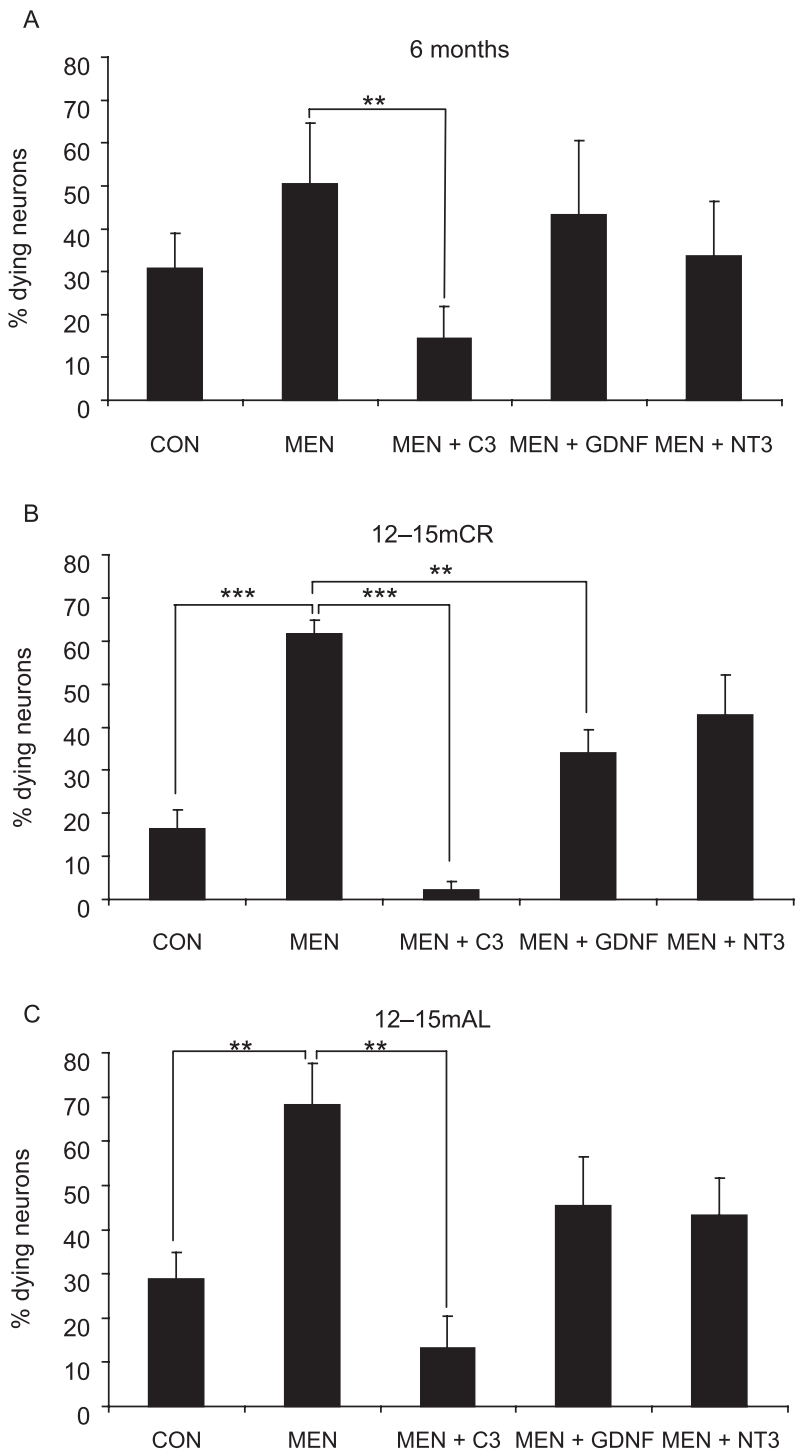

Fig. 5 Rescue by antioxidants and neurotrophic factors of ROS-induced cell death. Bar charts showing effects of menadione, with and without pretreatment with antioxidants (C3) and neurotrophic factors (GDNF, NT3), on death of myenteric neurons from 6-month (A), 12-15mCR (B) and $12-15 \mathrm{mAL}(\mathrm{C})$ animals. Note that $\mathrm{C} 3$ reduces cell death at all ages very substantially, confirming that menadione induces cell death by raising intracellular ROS. NT3 has no significant effect on ROS levels in any of the experimental groups. In contrast, GDNF produces a significant (50\%, $P<0.01)$ reduction of menadione induced cell death in $12-15 \mathrm{mCR}$ preparations, but not in age-matched AL preparations. All groups, $(n=4)$, except 6 months MEN + GDNF, and 12-15mCR MEN + C3, $(n=3)$.

outset, we wished to establish more accurately the timescale of neuron loss in order that examination of vulnerability to cell death could be studied before cell death had occurred, and also to consider the relation of age-related neuron cell loss to other aspects of the aging process. Previously, neuron loss has been shown to occur around 16 months of age (Cowen et al., 2000); here we demonstrate that it develops rapidly between 12 and 13 months and seems to be complete by 17 months in AL-fed rats as demonstrated by the HuC/D results. Although rats and 
mice remain relatively healthy at 12 months of age, it is thought that these rodents rarely live longer than 12 months in the wild (Phelan \& Austad, 1989). Selective pressures on neuroprotective mechanisms are therefore likely to be reduced or nonexistent by this age, providing a possible explanation for this apparently early and substantial neuron loss. Through the use of alternative markers (PGP 9.5, HuC/D) and staining methods (immunofluorescence, toluidine blue staining of resin-embedded sections) we confirm that PGP 9.5 labelling provides an accurate indication of age-related neuron loss. Resin-embedded sections exhibited substantial 'holes' surrounded by enteric glial cells in ganglia from AL-fed, aged rats. This arrangement resembles the nodules of Nageotte seen in dorsal root ganglia following pathological neuron loss (Thomas et al., 1997).

Significant neuron loss is only seen in AL-fed animals at the 12- to 15-month age stage, confirming previous observations that diet is a key coregulator of age-related neuron cell death; however, a smaller but significant $(P<0.05)$ loss of neurons is seen in $C R$ animals at 24 months indicating the dietary neuroprotection is not complete. $C R$ is known to increase the expression of neuroprotective genes including those encoding protein chaperones, neurotrophic factors (Prolla \& Mattson, 2001), mitochondrial gene expression (Nicoletti et al., 2005) and repressors of apoptosis in the aging rat brain (Shelke \& Leeuwenburgh, 2003). Our data confirm the protective effect of dietary restriction against age-related neuron cell death. Furthermore, the observation that cellular vulnerability to aging occurs relatively early in life supports predictions based on the evolutionary theory of aging (Kirkwood \& Austad, 2000) that dietary restriction will enhance investment in cellular maintenance and repair during adult, reproductive life.

We examined the possibility that myenteric neurons expressing the calcium-binding proteins, calretinin and calbindin (Abalo et al., 2005), might be particularly vulnerable to age-related neurodegeneration as a result of calcium dyshomeostasis ( $\mathrm{Bu}$ et al., 2003), which may be linked to altered mitochondrial ROS generation in old age (Mattson \& Liu, 2002). Calretinin-positive neurons form a far larger (25\%) proportion of myenteric neurons compared to calbindin (2.5\%) but exhibited no agerelated loss. Calbindin-positive neurons, in contrast, exhibited a substantial $50 \%$ loss which was fully rescued by CR. This is an interesting addition to the literature on selective vulnerability of subpopulations of neurons in the enteric nervous system (Wade \& Cowen, 2004), but does not further our understanding of the contribution of calcium dyshomeostasis in this process, nor does it tell us more about the phenotype of the large majority of myenteric neurons which are vulnerable during aging.

Evidence is growing of an association between ROS and agerelated neurodegenerative disease (for review, see (Butterfield \& Lauderback, 2002; Poon et al., 2004). However, there are few direct demonstrations of increased ROS levels in the nondiseased aging nervous system, or of the protective effects of CR against a possible age-related increase in ROS (Poon et al., 2004). Indeed, in Drosophila a lack of correlation between mitochondrial ROS and lifespan was recently demonstrated
(Miwa et al., 2004). Our data show a significant increase in ROS levels in 12-15 months in both $A L$ and CR groups compared to 6-month myenteric neurons in vitro which is maintained in 24-month neurons. ROS levels were not markedly influenced by diet although the rate of increase in ROS levels at $1 \mathrm{~h}$ of incubation (following 20 min of loading with DHR, see Experimental procedures) was reduced in 12-15mCR neurons compared to the parallel AL group. ROS levels showed similar trends in both 24-month groups indicating that even after $50 \%$ of neurons had died in the $\mathrm{AL}$ group, the remainder continue to exhibit elevated ROS levels compared to young neurons. This suggests that while some neurons are more vulnerable than others to age-related neurodegeneration, all are exposed to the damaging effects of elevated ROS.

The free radical-sensing dyes have advantages and disadvantages (for review see Tarpey et al., 2004). An important advantage of DHR for the present study is its capacity to provide a clear, neuron-specific signal which allows us to separate the confounding effects of changes in ROS in smooth muscle, enteric glial and vascular endothelial cells, all of which contribute to the ROS signal. These considerations rule out the use of more widely accepted biochemical assays for ROS in our preparations. Our laboratory has recently published further methodology for the use of DHR in cell-specific ROS assays, including effects of age and growth factor treatments, which support the view that with appropriate controls this method can provide reliable information on superoxide generation (Jallali et al., 2005). Here, the possibility that the DHR signal is affected by alterations in ROS from sources other than superoxide (e.g. reactive nitrogen species) or by alterations in mitochondrial membrane potential (Hempel et al., 1999) was examined using the recently developed dye, HPF (Setsukinai et al., 2003; Ooe et al., 2005), which reacts specifically with hydroxyl radicals. We confirmed the principal DHR results (i.e. the effects of age and diet on ROS) by showing a significant age-related increase of over $50 \%$ in ROS levels in 12- to 15-month neurons which is partially reduced by $\mathrm{CR}$. Because HPF responds primarily to $\mathrm{OH}^{\bullet}$, derived principally from superoxide, this experiment indicates that DHR in this model is probably measuring a ROS signal predominantly originating in mitochondrial superoxide.

Neurotrophic factors have been recently shown to exert antioxidant effects in developing sympathetic neurons (Dugan et al., 1999); however, their role in the adult and aging nervous system has not been investigated. We demonstrate that two of the principal endogenously expressed neurotrophic factors in the gut, GDNF and NT3, reduce ROS levels significantly in 6 months myenteric neurons showing that neurotrophic factors have antioxidant effects in adult myenteric neurons. This neuroprotective role was abolished in older (12-15 months and 24 months) neurons by $A L$ feeding but retained with $C R$, showing that diet influences the capacity of neurotrophic factors to affect antioxidant defence in the adult nervous system. Interestingly, the effects of both GDNF and NT3 are maximal in young and CR-fed groups during the first hour of treatment and then plateau out between 1 and $2 \mathrm{~h}$. This result suggests that neurotrophic factors 
have a finite effect on ROS homeostasis, but does not indicate whether they influence ROS generation by direct effects on mitochondrial function or through the up-regulation of antioxidants. Further experiments are required to examine this question.

ROS are increasingly understood to be associated with neurodegeneration, including neuron cell death, in a number of pathological conditions (see Introduction). However, while age is generally the strongest predictor of neurodegenerative disease, there is little evidence that nonpathological aging is accompanied by ROS-induced neuronal cell death. Recent data (Cowen et al., 2000) demonstrated that rat myenteric neurons are exceptionally vulnerable to cell death with over $50 \%$ of cells dying in animals at a relatively early stage in the aging process $(16 \mathrm{~m})$. Because cell death under these circumstances, even in relatively short-lived animals, occurs over weeks and months it is not possible to observe cell death directly in vivo or in ex vivo preparations. Stimulating the mitochondrial electron transport chain using the redox-cycling drug, menadione, causes oxidative stress and, in some cells, apoptotic cell death (Laux \& Nel, 2001; Gerasimenko et al., 2002). We therefore exposed myenteric neurons to menadione in vitro in order to examine their vulnerability to ROS-induced apoptotic cell death. TUNEL-positive neurons increased significantly following menadione treatment in 12- to 15-month and 24-month neurons from AL-fed animals in comparison with neurons from 6-month animals. The proportional increases in apoptotic neurons were $21 \%$ and $43 \%$, respectively, while in contrast only $3 \%$ of 6 -month neurons responded in the same way. Interestingly, CR very largely protected neurons against ROS-induced apoptotic cell death since the difference between the proportions of apoptotic neurons in $12 \mathrm{mCR}$ and 6 -month was not statistically significant. In addition to the characteristic brown nuclear staining characteristic of the TUNEL reaction, many dying neurons exhibited fragmented and pyknotic nuclei, indicating that they were indeed undergoing apoptotic cell death. In addition, other cells including smooth muscle, endothelial and glial cells exhibited widespread vulnerability to ROS-induced apoptosis.

Stimulation of ROS formation from the mitochondrial electron transport chain therefore provokes apoptotic cell death in older adult and aged neurons. Furthermore, like the ROS levels seen in vitro in these neurons, ROS-induced apoptotic neuron cell death is sensitive to diet, being largely prevented by CR at 12-15 months (Fig. 4 and above) as well as at 24 months (data not shown). This is the first demonstration that, during nonpathological aging, neurons become vulnerable to ROSinduced apoptosis and that CR plays a key role in protecting neurons against this form of stress-induced cell death.

Adult neurons become relatively independent of neurotrophic factors for their survival in vitro, as has been demonstrated by studies of adult sensory (Vogelbaum et al., 1998) and sympathetic (Easton et al., 1997; Orike et al., 2001b) neurons. NGF-independent survival of adult sympathetic neurons appears to be the result of an intrinsic up-regulation of PI3-kinase signalling (Orike et al., 2001a), which may be set by early life exposure to varying levels of neurotrophic factors (Gatzinsky et al., 2004) (T. Cowen \& M. Campioni-Noack, unpublished observations). However, this observation does not rule out the possibility that ongoing neurotrophin signalling influences in an unknown way the responses of adult and aging neurons to oxidative stress, hence protecting them against ROS-induced cell death. We therefore attempted to discover whether menadioneinduced death of neurons could be ameliorated by in vitro treatment with antioxidants and by neurotrophic factors. The synthetic antioxidant carboxyfullerene, C3, reduced the extent of cell death very significantly in young and 12 - to 15 -month groups, with greatest effect in the $12-15 \mathrm{mCR}$ group, indicating that menadione-induced cell death was indeed the result of elevated ROS. Pretreatment with GDNF resulted in significant protection from menadione-induced cell death in the $12-15 \mathrm{mCR}$ group only, confirming our hypothesis from the ROS experiments that $C R$ enhances antioxidant defence by neurotrophic factors. In contrast, NT3 effected a small but nonsignificant rescue of 12$15 \mathrm{mCR}$ neurons, despite the fact that it significantly depressed ROS levels in similar samples. The lack of protective effect of GDNF on menadione-induced cell death in young neurons, combined with the significant effect of C3, may be explained by the presence of optimal levels of intrinsic GDNF and saturated neuronal responses in the young myenteric plexus. Further studies of the relation between specific neurotrophic factors, antioxidant enzyme activity and cell death pathways are underway in attempts to resolve these apparent anomalies.

In summary, our results confirm the selective loss of rat myenteric neurons during aging and suggest a plausible mechanism whereby age-related neuron cell death is induced by elevated intraneuronal levels of ROS, which are accentuated by ad libitum feeding. In contrast, dietary restriction protects against ROSinduced neuronal cell death through mechanisms which include enhancement of a novel, antioxidant defensive role of neurotrophic factors.

\section{Experimental procedures}

\section{Animals}

All experiments were carried out on male Sprague Dawley rats from a colony maintained at Royal Free and University College Medical School (RFUCMS). All animals were reared up to 6 months of age with ad libitum (AL) access to BEEKAY Rat and Mouse standard 1 diet (B\&K Universal Ltd, Hull, UK), composed of $19 \%$ crude protein, $5 \%$ oil, $4 \%$ fibre and $72 \%$ dry matter. The caloric content is $16.16 \mathrm{~mJ} / \mathrm{kg}$ and the rats consumed an average of 50-60 g per day (i.e. $0.80-0.96 \mathrm{~mJ}$ per day). From 6 months of age, some animals were transferred to a calorierestricted diet (CR) of $25 \mathrm{~g}$ per day $(0.40 \mathrm{~mJ})$, whereas others remained on the $A L$ dietary regime. Numbers of samples used in each experiment are included in the figure legends.

Animals between the ages of 6 and 24 months were killed by carbon dioxide inhalation. Caloric restriction and all other conditions were undertaken in compliance with UK Home Office legislation under the Animals (Scientific Procedures) Act 1986. 


\section{Tissue preparation}

Immediately after respiration and heart beat ceased, the abdominal cavity was opened and the small intestine removed in toto.

\section{Dimensions of small intestine}

After the animals were killed, gut contents were flushed out with Hanks balanced salt solution (HBSS; pH 7.4). The gut was then inflated with HBSS to a pressure at which the gut began to curl (Johnson et al., 1998) and placed on a sheet of transparent plastic. Marks showing length and diameter were made at several points along the gut and transferred to graph paper allowing the total area of gut wall to be calculated.

\section{Numbers of neurons}

Ileum was removed from $A L$ and $C R$ rats at 6, 12, 13, 15 and 24 months of age, inflated with HBSS, fixed in 4\% paraformaldehyde for $2 \mathrm{~h}$, washed in PBS, and treated overnight with PBS $+20 \%$ sucrose. Strip preparations of the muscularis externa containing the myenteric plexus of neurons were dissected off, opened and sections taken from 10 to $20 \mathrm{~cm}$ of distal ileum. Samples were processed for immunocytochemistry using PGP 9.5 (UltraClone, Isle of Wight, UK), HuC/D (Molecular Probes) and calretinin (Swant Antibodies, Bellinzona, Switzerland) polyclonal antibodies and a monoclonal antibody against calbindin (Swant), with appropriate Alexafluor (Molecular Probes) secondary antibodies. PGP 9.5 and HuC/D are established as pan-neuronal markers for rodent myenteric neurons (Johnson et al., 1998; Phillips et al., 2004). Neurons were counted using a fluorescence microscope, expressed as numbers of neurons per $\mathrm{mm}^{2}$ and corrected for any age- or diet-related changes in size of the gut wall. All procedures have been described previously in detail (Johnson et al., 1998). To confirm that loss of PGP-immunoreactive neuron numbers coincided with neuron loss, some specimens were processed for resin embedding. One-micrometre sections were cut on an ultramicrotome, stained for toluidine blue and examined by light microscopy using a $\times 40$ objective.

\section{ROS levels in neurons}

An in vitro assay of ROS levels in myenteric neurons has been developed in our laboratory using the fluorescent probe, dihydrorhodamine (DHR; Molecular Probes) (Soubeyre et al., 2001; Jallali et al., 2005). Comparisons of DHR with another ROSsensitive probe, dichlorofluorescein diacetate (DCF-DA) (Hempel et al., 1999), demonstrated that the latter was not retained adequately in cells (Jallali et al., 2005). It should be borne in mind that these preparations contain many different cell types (muscle, connective tissue cells, endothelium) as well as neurons, hence more conventional biochemical assays for ROS are inappropriate.

Strip preparations of the myenteric plexus were prepared as before and transferred to F14 culture medium in 12-well culture plates maintained at $37^{\circ} \mathrm{C}$ in a $\mathrm{CO}_{2}$ incubator. Samples were treated with $10 \mu \mathrm{M}$ DHR for 20 min then maintained under the same conditions for a further 1-4 h. Graphs show the timescale starting from the point of incubation of the tissues in DHR (Fig. 3C-H). The first measurement was taken at 20 min when DHR loading was complete, termed time zero. Eighty- and 140min time points indicate 1 and $2 \mathrm{~h}$ of incubation post-probe loading, respectively. Preliminary experiments showed that responses peaked at $2 \mathrm{~h}$ (data not shown). To ensure that the changes in fluorescence signal obtained using DHR were not confounded by alterations in mitochondrial membrane potential, ferric iron or other age-associated variables (Hempel et al., 1999), comparisons were made between 6-month and 24-month AL preparations using the novel ROS-sensing dye, HPF (10 $\mu \mathrm{M}$; Alexis Biochemicals, Nottingham, UK) (Setsukinai et al., 2003), in samples pre-incubated for 20-30 min (as for DHR) then maintained for up to $3 \mathrm{~h}$ in F14 medium containing HPF following preliminary experiments showing that fluorescence was maximal at 2-3 h (data not shown).

\section{Free radical regulation}

In order to examine the role of neurotrophic factors in reducing free radical levels in enteric neurons from animals of different ages and subjected to different dietary regimes, duplicate samples were pretreated for $2 \mathrm{~h}$ with those neurotrophic factors with established roles in the developing and adult ENS (Chalazonitis et al., 2001; Busciglio et al., 2002), namely NT3, GDNF and IGF-1 at $100 \mathrm{ng} \mathrm{mL}^{-1}$. NGF was used at the same concentration as a control since it has no known role in the adult ENS. ROS-sensing dyes were added for the last 20-30 min of the pre-incubation.

\section{Imaging and image analysis}

At 0,1 and $2 \mathrm{~h}$ after pre-incubations, samples were removed, mounted in antifade mountant (Citifluor, UK) and imaged using confocal microscopy. Single 2 - $\mu$ m optical slices were obtained at a constant depth within the myenteric ganglia under strictly standardized conditions. Laser current was monitored and neutral density, aperture, black level, Kalman filtering and all other settings were maintained constant. A minimum of six images of non-overlapping ganglia containing a total of at least 50 neurons from each sample were stored and subjected to image analysis using a Zeiss Kontron image analysis system (KS400 V2/3, Carl Zeiss Ltd, Welwyn Garden City, UK). Nucleated profiles of neurons were selected and the neuronal perimeter traced interactively and filled to generate a mask. The masks of all traced neurons within a ganglion were then used to measure mean grey value (intensity) $\left(\mathrm{GV} / \mu \mathrm{m}^{2}\right)$ and integrated grey value per cell (GV/cell), as well as cell size.

\section{Apoptotic neuronal cell death}

Preparations similar to those used previously were treated with the redox-cycling drug, menadione (50 $\mu \mathrm{m}$ ) (lyanagi, 1990), for $4 \mathrm{~h}$. After treatment, samples were fixed in $4 \%$ paraformaldehyde for $2 \mathrm{~h}$, washed in PBS, followed by overnight treatment with 20\% sucrose in PBS, then frozen in cryoprotectant and cryosectioned at $7 \mu \mathrm{m}$. TUNEL staining of the sections was carried out using a kit (NeuroTACS II, TA900, R\&D Systems, UK) 
to demonstrate apoptotic cell profiles. Numbers of apoptotic neurons were scored on at least three sections per sample using a light microscope and $\times 40$ objective. Brown-stained neuronal nuclei, often exhibiting fragmentation, were scored as apoptotic, compared with blue-stained oval nuclei which were scored as normal. Visual analysis of neuronal nuclear morphology was carried out in parallel. Apoptotic neuron numbers were expressed as a percentage of normal neuron numbers.

To ensure that TUNEL staining provided an accurate indication of cell death, and because of the relatively limited sampling in cryosections of myenteric ganglia, similar preparations, but without fixation, were examined directly in the confocal microscope after treatment for 20 min using the Live-Dead assay following manufacturer's instructions (Molecular Probes). Neurons were distinguished from glial cells by clear differences of size and shape. Green, calcein-stained live neurons and red, ethidium-stained nuclei of dead neurons were counted in the same preparations and numbers of dead cells were expressed as a proportion of the total (live + dead).

\section{Protection by neurotrophic factors against menadione-induced cell death}

Because the Live-Dead assay gave better sampling and as a result more reliable quantification compared to TUNEL staining, it was used to study the protective effects of neurotrophic factors on menadione-induced cell death. Similar preparations to those used previously were exposed to GDNF and NT3 (100 $\mathrm{ng} \mathrm{mL}^{-1}$, as before) and then to menadione (50 $\mu \mathrm{m}$, as before). After $4 \mathrm{~h}$, cells were treated with the reagents of the Live-Dead assay, imaged and the proportion of dead cells counted, as previously described.

\section{Data analysis}

Mean, standard deviations and standard errors of mean were calculated using MS Excel. Statistical analysis of variance was carried out using two-way analysis of variance (ANOVA) followed by post-hoc LSD or Bonnferoni tests of significant differences between data pairs with $P<0.05$ assigned as statistically significant (Unistat or Graphpad software).

\section{Acknowledgments}

We acknowledge support from the BBSRC SAGE Initiative, project no.108/SAG10013. We thank Dr Laura Dugan for supplying C3, and are grateful for skilled assistance from Innis Clatworthy in preparing resin-embedded sections and from Clare Underwood with photography. We thank Dr Brian Merry for helpful discussion of the manuscript.

\section{References}

Abalo R, Jose RA, Vera G, Isabel MM (2005) Ileal myenteric plexus in aged guinea-pigs: loss of structure and calretinin-immunoreactive neurones. Neurogastroenterol. Motil. 17, 123-132.
Baquet ZC, Gorski JA, Jones KR (2004) Early striatal dendrite deficits followed by neuron loss with advanced age in the absence of anterograde cortical brain-derived neurotrophic factor. J. Neurosci. 24, 4250-4258

Bu J, Sathyendra V, Nagykery N, Geula C (2003) Age-related changes in calbindin-D28k, calretinin, and parvalbumin-immunoreactive neurons in the human cerebral cortex. Exp. Neurol. 182, 220-231.

Busciglio J, Pelsman A, Wong C, Pigino G, Yuan M, Mori H, Yankner BA (2002) Altered metabolism of the amyloid beta precursor protein is associated with mitochondrial dysfunction in Down's syndrome. Neuron. 33, 677-688.

Butterfield DA, Lauderback CM (2002) Lipid peroxidation and protein oxidation in Alzheimer's disease brain: potential causes and consequences involving amyloid beta-peptide-associated free radical oxidative stress(1,2). Free Radic. Biol. Med. 32, 1050-1060.

Chalazonitis A, Pham TD, Rothman TP, Distefano PS, Bothwell M, Blair-Flynn J, Tessarollo L, Gershon MD (2001) Neurotrophin-3 is required for the survival-differentiation of subsets of developing enteric neurons. J. Neurosci. 21, 5620-5636.

Cowen T, Gavazzi I (1998) Plasticity in adult and ageing sympathetic neurons. Prog. Neurobiol. 54, 249-288.

Cowen T, Johnson RJR, Soubeyre V, Santer RM (2000) Restricted diet rescues rat enteric motor neurones from age related cell death. Gut 47, 653-660.

Cowen T, Woodhoo A, Sullivan CD, Jolly R, Crutcher K, Wyatt S, Michael GJ, Orike N, Gatzinsky KP, Thrasivoulou C (2003) Reduced age-related plasticity of neurotrophin receptor expression in selected sympathetic neurons of the rat. Aging Cell 2, 59-70.

Dugan LL, Creedon DJ, Johnson EM, Holtzman DM (1999) Rapid suppression of free radical formation by nerve growth factor involves the mitogen-activated protein kinase pathway. Proc. Natl Acad. Sci. U S A 94, 4086-4091.

Dugan LL, Du Turetsky DMC, Lobner D, Wheeler M, Almli CR, Shen CK, Luh TY, Choi DW, Lin TS (1997) Carboxyfullerenes as neuroprotective agents. Proc. Natl Acad. Sci. U S A 94, 9434-9439.

Easton RM, Deckwerth TL, Parsadanian AS, Johnson EM Jr (1997) Analysis of the mechanism of loss of trophic factor dependence associated with neuronal maturation: a phenotype indistinguishable from Bax deletion. J. Neurosci. 17, 9656-9666.

Finkel T, Holbrook NJ (2000) Oxidants, oxidative stress and the biology of ageing. Nature 408, 239-247.

Gabella G (1989) Fall in the number of myenteric neurons in aging guinea pigs. Gastroenterology 96, 1487-1493.

Gatzinsky KP, Thrasivoulou C, Campioni-Noack M, Underwood C, Cowen T (2004) The role of NGF uptake in selective vulnerability to cell death in ageing sympathetic neurons. Eur. J. Neurosci. 20, 28482856.

Gerasimenko JV, Gerasimenko OV, Palejwala A, Tepikin AV, Petersen OH, Watson AJ (2002) Menadione-induced apoptosis: roles of cytosolic $\mathrm{Ca}(2+)$ elevations and the mitochondrial permeability transition pore. J. Cell Sci. 115, 485-497.

Hempel SL, Buettner GR, O'Malley YQ, Wessels DA, Flaherty DM (1999) Dihydrofluorescein diacetate is superior for detecting intracellular oxidants: comparison with 2', $7^{\prime}$-dichlorodihydrofluorescein diacetate, 5 (and 6) -carboxy-2', $7^{\prime}$-dichlorodihydrofluorescein diacetate, and dihydrorhodamine 123. Free Radic. Biol. Med. 27, 146-159.

Huang X, Moir RD, Tanzi RE, Bush Al, Rogers JT (2004) Redox-active metals, oxidative stress, and Alzheimer's disease pathology. Ann. N. Y. Acad. Sci. 1012, 153-163.

lyanagi T (1990) On the mechanism of one-electron reduction of quinones by microsomal flavin enzymes: the kinetic analysis between cytochrome B5 and menadione. Free Radic. Res. Commun. 8, 259-268. 
Jallali N, Ridha H, Thrasivoulou C, Underwood C, Butler PE, Cowen T (2005) Vulnerability to ROS-induced cell death in ageing articular cartilage: the role of antioxidant enzyme activity. Osteoarthritis Cartilage 13, 614-622.

Johnson RJR, Schemann M, Santer RM, Cowen T (1998) The effects of age on the overall population and on subpopulations of myenteric neurons in the rat intestine. J. Anat. 192, 479-488.

Jung C, Rong Y, Doctrow SR, Baudry M, Malfroy B, Xu Z (2001) Synthetic superoxide dismutase/catalase mimetics reduce oxidative stress and prolong survival in a mouse amyotrophic lateral sclerosis model. Neurosci. Lett. 304, 157-160.

Kirkwood TB, Austad S (2000) Why do we age? Nature 408, 233-238.

LaFerla FM (2002) Calcium dyshomeostasis and intracellular signalling in Alzheimer's disease. Nat. Rev. Neurosci. 3, 862-872.

Lau FC, Shukitt-Hale B, Joseph JA (2005) The beneficial effects of fruit polyphenols on brain aging. Neurobiol Aging 26 (Suppl. 1), 128-132.

Laux I, Nel A (2001) Evidence that oxidative stress-induced apoptosis by menadione involves Fas-dependent and Fas-independent pathways. Clin. Immunol. 101, 335-344.

Mattson MP, Chan SL, Duan W (2002) Modification of brain aging and neurodegenerative disorders by genes, diet, and behavior. Physiol. Rev. 82, 637-672.

Mattson MP, Liu D (2002) Energetics and oxidative stress in synaptic plasticity and neurodegenerative disorders. Neuromolecular Med. 2, 215-231.

Miller FD, Kaplan DR (2001) On Trk for retrograde signaling. Neuron. 32, 767-770.

Mills EM, Takeda K, Yu ZX, Ferrans V, Katagiri Y, Jiang H, Lavigne MC, Leto TL, Guroff G (1998) Nerve growth factor treatment prevents the increase in superoxide produced by epidermal growth factor in PC12 cells. J. Biol. Chem. 273, 22165-22168.

Miwa S, Riyahi K, Partridge L, Brand MD (2004) Lack of correlation between mitochondrial reactive oxygen species production and life span in Drosophila. Ann. N. Y. Acad. Sci. 1019, 388-391.

Morrison JH, Hof PR (1997) Life and death of neurons in the aging brain. Science 278, 412-419.

Nicoletti VG, Marino VM, Cuppari C, Licciardello D, Patti D, Purrello VS, Stella AM (2005) Effect of antioxidant diets on mitochondrial gene expression in rat brain during aging. Neurochem. Res. 30, 737752.

Nunomura A, Perry G, Aliev G, Hirai K, Takeda A, Balraj EK, Jones PK, Ghanbari H, Wataya T, Shimohama S, Chiba S, Atwood CS, Petersen RB, Smith MA (2001) Oxidative damage is the earliest event in Alzheimer disease. J. Neuropathol. Exp. Neurol. 60, 759767.

Ooe H, Taira T, Iguchi-Ariga SMM, Ariga H (2005) Induction of reactive oxygen species by bisphenol $A$ and abrogation of bisphenol A-induced cell injury by DJ-1. Toxicol. Sci. 88, 114-126.

Orike N, Middleton G, Buchman VL, Cowen T, Davies AM (2001a) Role of PI 3-kinase, Akt and Bcl-2-related proteins in sustaining the survival of neurotrophic factor-independent adult sympathetic neurons. J. Cell Biol. 154, 995-1005.

Orike N, Thrasivoulou C, Wrigley A, Cowen T (2001b) Differential regulation of survival and growth in adult sympathetic neurons: an in vitro study of neurotrophin responsiveness. J. Neurobiol. 47, 295305.
Phelan JP, Austad SN (1989) Natural selection, dietary restriction, and extended longevity. Growth Dev. Aging 53, 4-6.

Phillips RJ, Hargrave SL, Rhodes BS, Zopf DA, Powley TL (2004) Quantification of neurons in the myenteric plexus: an evaluation of putative pan-neuronal markers. J. Neurosci. Meth. 133, 99-107.

Phillips RJ, Kieffer EJ, Powley TL (2003) Aging of the myenteric plexus: neuronal loss is specific to cholinergic neurons. Auton. Neurosci. 106, $69-83$.

Poon HF, Calabrese V, Scapagnini G, Butterfield DA (2004) Free radicals and brain aging. Clin. Geriatr. Med. 20, 329-359.

Porter AJ, Wattchow DA, Brookes SJH, Schemann M, Costa M (1996) Choline acetyltransferase immunoreactivity in the human small and large intestine. Gastroenterology 111, 401-408.

Prolla TA, Mattson MP (2001) Molecular mechanisms of brain aging and neurodegenerative disorders: lessons from dietary restriction. Trends Neurosciences 24, S21-S31.

Salinas M, Diaz R, Abraham NG, Ruiz de Galarreta CM, Cuadrado A (2003) Nerve growth factor protects against 6-hydroxydopamineinduced oxidative stress by increasing expression of heme oxygenase1 in a phosphatidylinositol 3-kinase-dependent manner. J. Biol. Chem. 278, 13898-13904.

Schapira AH (1998) Mitochondrial dysfunction in neurodegenerative disorders. Biochimica Biophysica Acta 1366, 225-233.

Setsukinai K, Urano Y, Kakinuma K, Majima HJ, Nagano T (2003) Development of novel fluorescence probes that can reliably detect reactive oxygen species and distinguish specific species. J. Biol. Chem. 278, 3170-3175.

Shelke RR, Leeuwenburgh C (2003) Lifelong caloric restriction increases expression of apoptosis repressor with a caspase recruitment domain (ARC) in the brain. FASEB J. 17, 494-496.

Sohal RS, Weindruch R (1996) Oxidative stress, caloric restriction, and aging. Science 273, 59-63.

Soubeyre V, Thrasivoulou C, Hoyle CH, Saffrey MJ, Cowen T (2001) Free radical induced cell death in ageing rat enteric neurons. Abstr. -Soc Neurosci 31, 169.

Tarpey MM, Wink DA, Grisham MB (2004) Methods for detection of reactive metabolites of oxygen and nitrogen: in vitro and in vivo considerations. Am. J. Physiol. Regul. Integr. Comp. Physiol. 286, R431R444.

Thomas PK, Landon DN, King RHM (1997) Diseases of peripheral nerves. In Greenfield's Neuropathology (Graham DI, Lantos PL, eds). London: Arnold, pp. 367-432.

Tsao N, Luh T-Y, Chou C-K, Chang TY, Wu J-J, Liu C-C, Lei H-Y (2002) In vitro action of carboxyfullerene. J. Antimicrob Chemother 49, 641649.

Turlejski K, Djavadian R (2002) Life-long stability of neurons: a century of research on neurogenesis, neuronal death and neuron quantification in adult CNS. Prog. Brain Res. 136, 39-65.

Vogelbaum MA, Tong JX, Rich KM (1998) Developmental regulation of apoptosis in dorsal root ganglion neurons. J. Neuroscience $\mathbf{1 8 , 8 9 2 8 -}$ 8935.

Wade PR, Cowen T (2004) Neurodegeneration: a key factor in the ageing gut. Neurogastroenterol. Motil. 16, 19-23.

Young HM, Bergner AJ, Mü Ller T (2003) Acquisition of neuronal and glial markers by neural crest-derived cells in the mouse intestine. J. Comp. Neurol. 456, 1-11. 